\title{
The vitality of death discussions. Comments on Bacigalupo et al., 'The debate about death: an imperishable discussion?'
}

\author{
ALEJANDRO SERANI-MERLO*,1
}

\author{
*Universidad de los Andes \\ ${ }^{1}$ Corresponding author San Carlos de Apoquindo 2200, Las Condes, Santiago, Chile \\ Phone 56-2-4129464 Email aserani@uandes.cl
}

Imperishable or not, the debate about death, seems to be quite alive. This is at least what Bacigalupo, Huerta and MontefuscoSiegmund intend to show in a stimulating review ${ }^{1}$. The discussion of every issue raised in this quite comprehensive review is beyond the scope of this commentary. We will focus then, only on a single subject: 'The problem of integration and the criteria of death'. More specifically, we would like to contend the proposed role of the Nervous System in somatic or physiological integration.

\section{EPISTEMOLOGICAL REMARKS}

In line with a general idea, -firstly proposed by the group of Darmouth Medical School in 1981, in order to clarify discussions about death ${ }^{2}-$ we distinguish three different epistemological levels of analysis: 1) A philosophico-metaphysical level of discussions, whose scope is to reflect on the nature, essence, idea, notion or definition of death; 2) A philosophicobiological level in which a certain number of general but concrete criteria of death are proposed; 3) A biomedical level of discussions in which an effort is done in order to determine verifiable empirical signs of the occurrence of the criteria and to develop the corresponding operative certificatory tests.

\footnotetext{
1 Bacigalupo F, Huerta D, Montefusco-Siegmund R The debate about death: an imperishable discussion? Biological Research ...

2 Bernat J, Culver C, Gert B Definition of death. Ann Intern Med 95: 652.1981.
}

At the biophilosophical level two main criteria of death have been proposed in recent discussions: i. The irreversible loss of the integration of the organism as a whole. ii. The permanent cessation of consciousness. The first criterion -usually referred as the biological criterion- is mainly sustained by physicians or biologically formed authors. The second one, -usually referred as the psychological criterion- is mainly sustained by philosophers and psychologically trained authors.

TWO WAYS OF UNDERSTANDING THE BIOLOGICAL CRITERIA

Until 1968, there was a tacit general agreement that certification of irreversible cardio-respiratory arrest was an inequivocal proof of the loss of the integration of the organism as a whole. Certification of the actual empirical occurrence of the criterion was equated with certification that death has occurred, and irreversible cardio-respiratory arrest was the proof for that. Since Harvard's Report of the Ad Hoc Committee to Examine the Definition of Brain Death ${ }^{3}$, however, a concurrent rationale for the actual occurrence of the criterion was developed. The Committee argued that irreversible cardio-respiratory arrest was

\footnotetext{
Special Communication, A Definition of Irreversible Coma, Report of the Ad Hoc Committee of the Harvard Medical School to Examine the Definition of Brain Death, JAMA 205 (6): 337-340.
} 
no longer a proof or a universally valid empirical fact for declaring the actual occurrence of the criterion. Irreversible coma due to whole brain irreversible damage was then proposed as the new rationale. The basis for this redefinition was that irreversible damage of the brain and not irreversible cardio-respiratory arrest verifies the loss of the unified functioning of the organism as a whole. Implicit or explicit in this argumentation was that the functioning of the nervous system is what permits unified action of the organism as a whole, mainly through the action of brain stem vasopressor and respiratory neurons. As Bacigalupo et al. show in their paper, the idea that it is the proper action of the Nervous System what integrates or unifies somatic or physiological functioning, has been considered since then by many, as a self evident biological fact.

\section{IS PHYSIOLOGICAL INTEGRATION REALLY A NERVOUS FUNCTION?}

As Bacigalupo et al. mention, the neurologist Alan Shewmon has extensively published arguing against the attribution to the Nervous System of a role of somatic integrator. Shewmon has in fact proposed empirical and theoretical arguments tending to prove that Nervous System's role is not the one of being a physiological integrator ${ }^{4}$. Published reactions against Shewmon's arguments reveal -in our view- a generalized lack of clarity about what the Nervous System really does in the organism as a whole.

In two previous articles in Biological Research ${ }^{5} 6$ we have tried to precise the real place of Nervous System's actions in

Shewmon A 'Brain Body' Disconnection: Implications for the Theoretical Basis of 'Brain Death', In: de Mattei R. (editor) "Finis Vitae, Is brain death still life ? Consiglio nazionale delle Ricerche/Rubettino (Siveria Mannelli) 2006.

5 Serani-Merlo A, Jaime Alvarez and the case against slow axoplasmic transport: epistemological reflections Biological Research 34: 111-115, 2001.

6 Serani-Merlo A, Paz R, Castillo A, The 'whole-animal approach' as a heuristic principle in neuroscience research Biological Research 38: 359-364, 2005. whole-animal phenomenology. We think that this theoretical framework could shed light on brain death discussions.

Three hierarchically interrelated kinds of functions are recognized in the Nervous System's actions: 1) autopoietic; 2) autonomic and 3) behavioural functions. Autopoietic-metabolic activity is common to all parts of the organism and its existence precedes - phylogenetically and ontogenetically- any specific nervous function. Behaviour -which embraces cognitive, affective and executive subfunctions- is essentially a variable activity which imposes changing demands upon autopoietic metabolism. Autonomic nervous function must in consequence match variable behavioural demands with variable but limited metabolic resources. Its role could be described then as regulatory or modulatory of metabolic activities in regard to behavioural requirements.

In mammalian intrauterine life, autopoietic metabolic activity chronologically precedes the existence of autonomic and behavioural functioning. Autopoietic metabolic activity of the organism as a whole is in fact the result of a very complex set of integrated activities. As Shewmon has argued, organic metabolic activity is in itself integrated, without a requirement for the living being of a special 'integratory' function. This is what makes living beings what they are, in contradistinction with machines. Autonomic and behavioural functioning appear then, when autopoietic metabolic activity is already long time integrated.

During intra-uterine life, mammals oxygenate their blood through maternal blood exchange of gases. In extra-uterine life ventilation assures this exchange depending on the indemnity of respiratory centre neurons in the brainstem. Severe injury to brainstem respiratory neurons impedes gas exchange, leading to a disruption of autopoietic metabolic integrated activity, in the same way as cardiac arrest does. Gas exchange and circulation -and not nervous system's function- are then critical or vital functions for the maintenance of autopoietic metabolic integrity. 
BRAIN, CARDIAC OR MERE DEATH?

As Bacigalupo et al. rightly point out; intensive care units have nowadays dramatically modified survival possibilities of injured human beings. Circulation and ventilation can be technically assisted, in order to eventually prevent autopoietic metabolic disruption or loss of the unitary functioning of the organism as a whole.

Circulatory or ventilatory arrest are not in themselves what verifies the criterion of death, they are the causes of autopoietic metabolic disruption. Artificially assisted organisms are still alive if signs of metabolic integration are still present. They are not organisms in a state of artificial life, they are naturally living technically assisted organisms.

Death or loss of the integrated functioning of the organism as a whole is then, the consequence of cardiac or ventilatory arrest; but neither irreversible cardiac arrest nor permanent damage to brainstem respiratory neurons are death in themselves.

Arguing physiologically, we contend then, that all patients in irreversible coma are already dead. In the actual state of the medical art most of them are in an irreversible process of dying, temporarily arrested by artificial technical means. Cardiac death or brain death, are actually misleading shorthands. Death is merely death. 


